\title{
Challenges of Capturing Design Rationales in Enterprise Architecture: A case study
}

\author{
Georgios Plataniotis ${ }^{1,2,4}$, Sybren de Kinderen ${ }^{3,4}$, and Henderik A. Proper ${ }^{1,2,4}$ \\ 1 Public Research Centre Henri Tudor, Luxembourg, Luxembourg \\ ${ }^{2}$ Radboud University Nijmegen, Nijmegen, the Netherlands \\ 3 University of Luxembourg, Luxembourg \\ 4 EE-Team, Luxembourg, Luxembourg ${ }^{\star \star}$ \\ \{georgios.plataniotis, erik.proper\}@tudor.lu, sybren.dekinderen@uni.lu
}

\begin{abstract}
In earlier work we introduced an approach that aims in the understanding of the reasoning that lies behind Enterprise Architecture (EA) designs. This EA rationalization approach helps uncover design constraints, follow good practices while avoid bad ones, and more. However, up to now, our assumptions regarding the usefulness of our approach were largely theory based.

In this paper, we use a real life case study in a Luxembourgish Research and Technology Organization (RTO) to identify from a practical point of view why EA rationalization is useful. Using a business process on budget forecasting as a focal point, firstly we analyze what has been designed. Thereafter, we analyze the reasoning behind these designs, and show how this reasoning is not expressed with current EA design techniques. Finally, we derive a set of challenges that an EA rationalization approach should face up to.
\end{abstract}

Keywords: Enterprise Architecture, Design Rationale, Design Decisions, Case Study

\section{Introduction}

Increasingly enterprises are faced with change due to challenges such as acquisitions, mergers, technological innovation and the introduction of new business processes. Enterprise Architecture (EA) is positioned as an instrument for helping enterprises to cope with such a change $[1,2]$. EA provides a toolset for analyzing the wide enterprise impact of a change, considering an enterprise holistically. As such, EA interrelates different perspectives on an enterprise [1]: strategic motivations, business processes, IT applications, to name a few. In so doing, EA instrument can be employed to, for example, reason about the impact-of-change

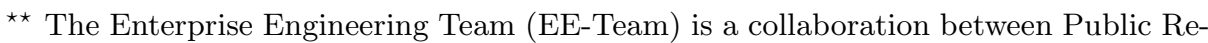
search Centre Henri Tudor, Radboud University Nijmegen and HAN University of Applied Sciences (www.ee-team.eu)
} 
of an IT application landscape [3], the enterprise wide impact of changing access control concerns [4] and more.

However, while EA frameworks and languages aid first in designing a change and subsequently implementing it, the reasons behind the design (hereafter referred to as design rationalization) are often left implicit. As a result, new designs are constructed in an ad-hoc manner [5], without taking into consideration constraints implied by past design decisions. Furthermore a survey amongst EA practitioners [6] suggests the relevance of design rationalization in motivating design decisions and for architectural maintenance. However, the same survey shows that practitioners often forego a structured approach for architectural rationalization. They largely capture design reasoning in an ad hoc manner, not structurally paying attention to rationalization concepts such as design alternatives, design criteria, and more.

As a result, our earlier work [7] introduces the EA anamnesis approach for rationalizing EA designs. EA anamnesis captures the decision making process behind an EA design, specifying design alternatives, design criteria, involved stakeholders, and more. Furthermore, EA anamnesis allows for capturing the observed impact of a design some time after the design decisions have been taken. As a result, we claim that EA anamnesis not only helps in uncovering the reasoning behind a design (by examining its decision making process), but also in analyzing the impacts of a design by comparing the initially taken design decisions to the impact that these decisions have.

However, up to now, the empirical evidence for the usefulness of EA anamnesis is limited to a survey amongst EA practitioners [6]. Although this survey provides first indications on the usefulness of EA rationalization, however it provides no in depth insight. For example: the survey elicits that a majority of the target population considers rationalization concepts such as "motivation", and "observed impact" as useful, but it does not provide any indication of why these are considered useful. Furthermore, as stated, the survey indicates that practitioners rationalize EA in an ad hoc way, without though stating what it is that they do not capture.

In this paper, we discuss a case study in a Luxembourgish Research and Technology Organization (RTO) for a more in depth analysis of the practical usefulness of EA rationalization. Furthermore, we identify rationalization requirements from practice to which our rationalization approach should respond. As such, the specific contribution is twofold: (1) to provide in depth evidence from a real life case study on the usefulness of EA rationalization, (2) to identify objectives from practice with which EA anamnesis, our rationalization approach, should comply.

Note that while the EA language ArchiMate 2.0 [8] has a motivational layer, it lacks concepts important for rationalization such as considered alternatives, decision criteria et cetera. As such, ArchiMate 2.0 is not a suitable language for architectural rationalization

This paper is structured as follows: Section 2 discusses the case study setup and the actual case study description. Section 3 discusses the challenges and 
the objectives for the development of rationale man agent system for enterprise architecture and Section 4 presents a set of concrete research questions which aim to address the mentioned objectives. Finally Section 5 concludes.

\section{Research and Technology Organization Case Study}

\subsection{Case study setup}

The research planning of the case study as well as this section structure are based on guidelines for case study research [9]. The main objectives of this case is to identify the main challenges for the development of rationale management system for Enterprise Architecture. In order to identify these objectives we conducted interviews with the involved stakeholders both from the business as well as the IT domain of the organization. The purpose of these interviews was to understand how they addressed the enterprise architecture challenges from their own domain of responsibility, what were the most important design decisions for them and how they documented these design decisions. Moreover, stakeholders provided us with the documentation of the project. We analyzed this documentation, we extracted design decisions, and finally compared them with those that emerged from the interviews.

\subsection{Budget forecasting at a Research and Technology Organization}

This case study was conducted in a research and technology organization (RTO) in Luxembourg, describes the introduction of a new budget management business process and presents how this process was supported by information systems in the context of an enterprise architecture transformation.

During the last couple of years, the Luxembourgish government introduced more strict rules on the budget spending of academic and research institutions. This policy had to be incorporated by the academic-research institutions of the country, meaning that the institutions should be able to establish long term financial projection plans. This would give to institutions a better awareness regarding the availability of resources and in turn the planning of future projects and personnel.

The RTO where the case study was conducted, did not have an established business process for budget estimation. Stakeholders from the management side of the RTO had to design this new business process. Their initial objectives were that this business process should provide a clear view on human resources and projects coverage, an input for the future hiring plan, comparison between the forecasted and valuable budget, and in general robustness of the organization's financial data. Last but not least, a training for the users of this new business process should be organized.

\subsection{Enterprise Transformation}

In this section we describe the main artifacts that support the realization of the new business goal. For our description we are based on ArchiMate EA models. 
In the context of this paper though we describe how this business process is supported by IT artifacts, we do not present details on how this business process is executed.

\section{The as-is architecture}

Figure 1 presents the enterprise architecture before the incorporation of the new budget forecast business process. The RTO had already implemented business processes that supported business stakeholders with the management of projects, finance and human resources. These business processes were supported by the corresponding information systems.

In this part we describe briefly the new business process and information systems that were used in the next step of the transformation to support the

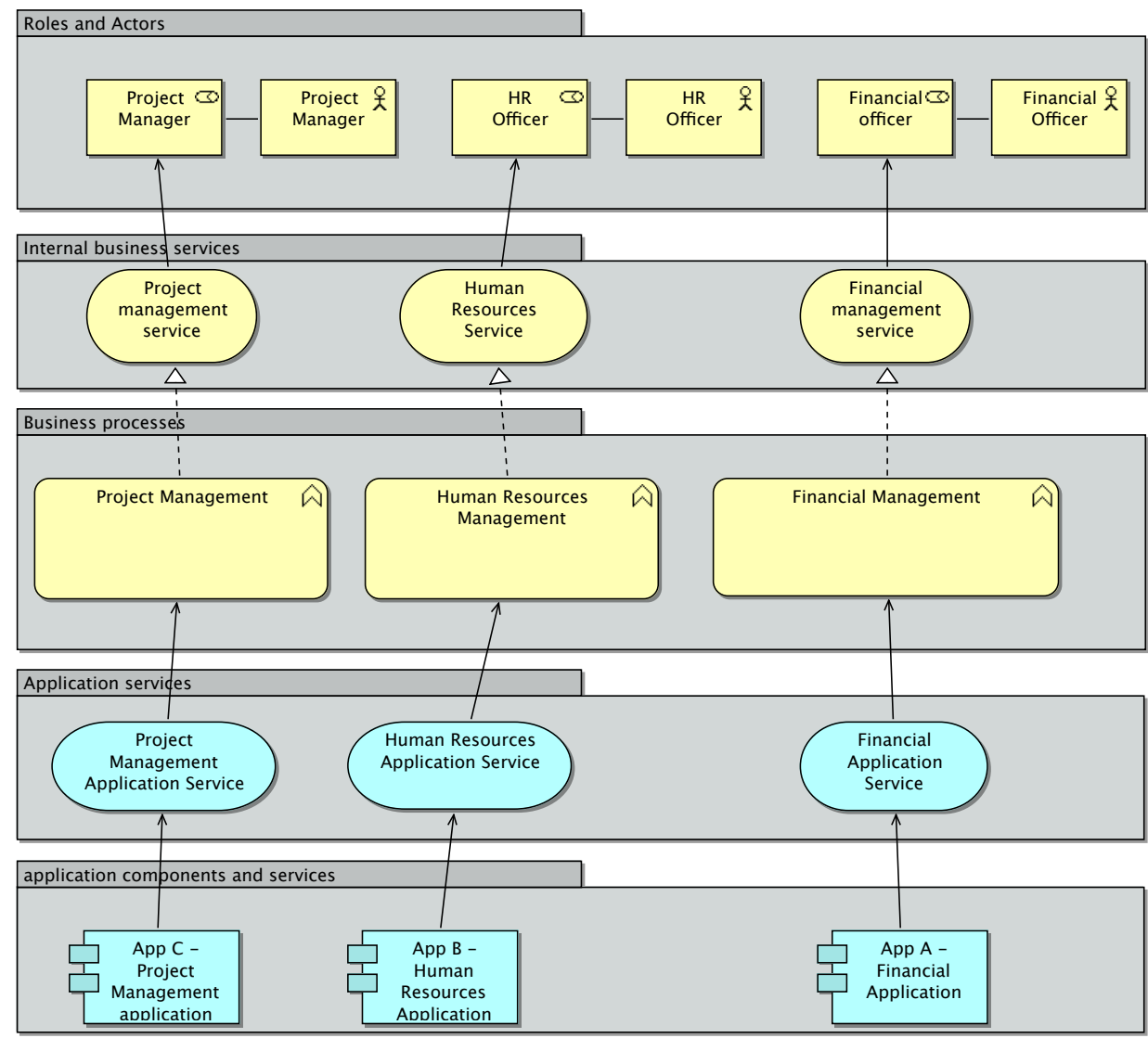

Fig. 1. The as-is architecture 
new business process.

Budget forecast business process: Here we describe the main characteristics of the budget forecast business process. The main objectives of this business process are the estimation and the planning of resources to ensure the planning activities, the assessment of the need for additional resources, the estimation of the associated budgets and the checking of the forecast in relation to the available budget in the RTO.

The budget forecast business process takes into account the major changes in exogenous or endogenous factors that significantly discredits the original budget estimate. In the case of the RTO these exogenous factors are the main external factor like refusal/acceptance of projects in calls and non-contracted benefits, while the endogenous maybe associated with personal assignments, change of strategy etc. The role of the business process is to provide annual budget estimates, which should be validated and approved by the finance department.

IT Systems: Application A is the main financial application of the organization. Main functionalities of this application is management of procurements, traveling costs, personal costs, overhead costs calculation, salaries payment and project dashboard. The access to this application in controlled and only allowed to financial officers.

Application B is the human resources management application. Tasks like resource allocation, start/end dated of work contracts, weekly calendar, different types of leaves (sickness, vacation etcetera) are executed by this application.

Application $\mathrm{C}$ is the project management application of the organization. The actual hours assigned per project in the organization are maintained in this application.

\section{To be architecture - First iteration}

Figure 2 depicts the EA model after the establishment of the budget forecast business process. From this model we can conclude that the business process was supported by the interaction and collaboration amongst Applications A, B, C and a spreadsheet application. However, due to some anomalies in the insertion of budget data through spreadsheets, stakeholders had to do some additional changes in the EA design.

\section{To-be architecture - Second iteration}

Figure 3 depicts a final iteration of the enterprise transformation. With this iteration stakeholders managed to addresses the above mentioned problem. Instead of using spreadsheets for entering the budget data, a new application interface was added in financial application A.

\subsection{Rationale behind the models}

In the previous section we described what was done in the enterprise architecture design in order to support the addition of the new budget forecast business 


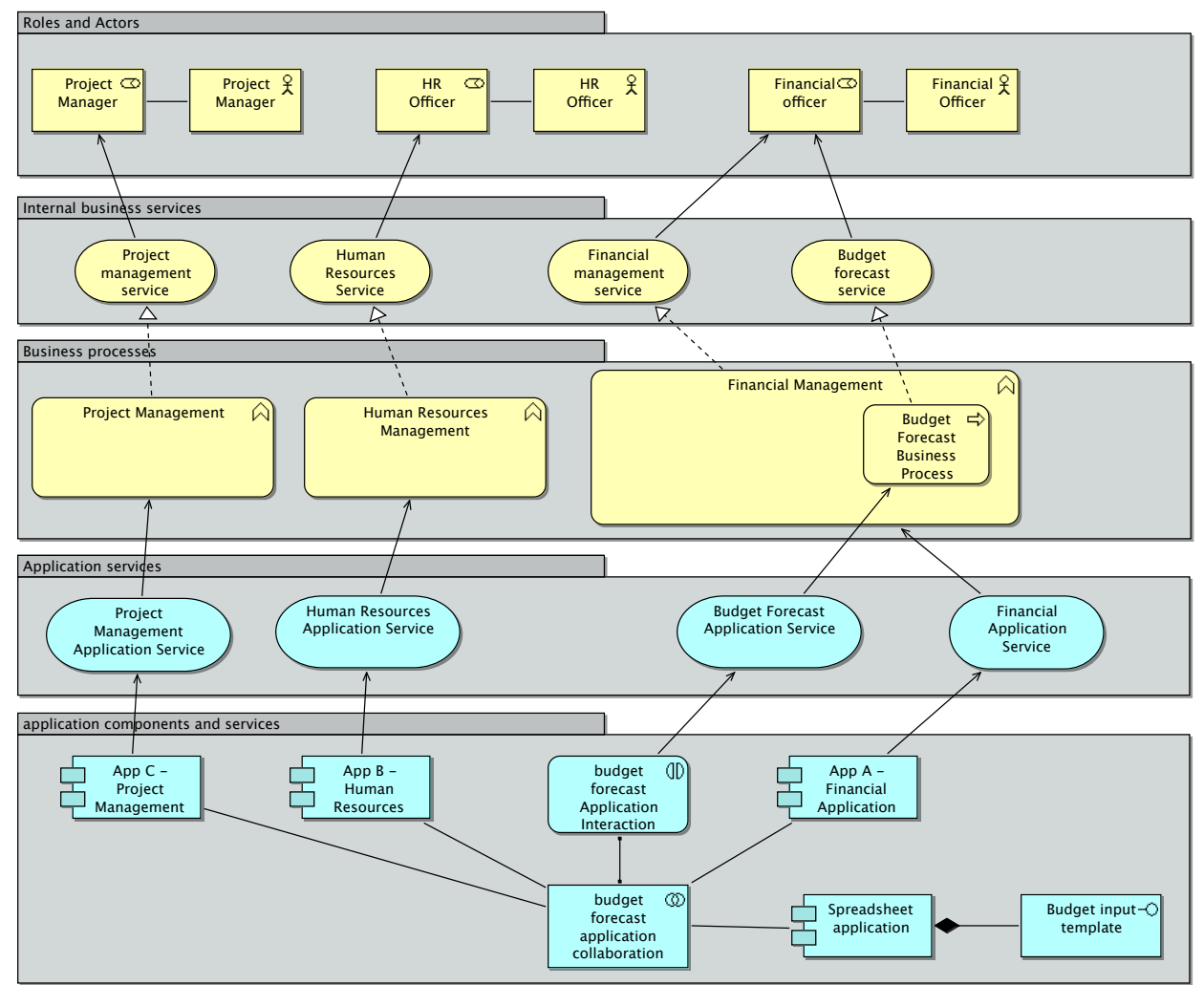

Fig. 2. To be architecture - First iteration

process. However, the reasons behind the realization of this designs are not captured by the EA models. As we mentioned before, ArchiMate motivational extension provides some reasoning behind the models, but mostly regarding the goal modeling domain. It can describe what were the drivers or requirements for an architectural change, but not the actual reasoning in terms of design decisions behind the model. Based on the case study we could potentially ask these questions:

Why these three IT systems were selected for the realization of the business process? Were there any other alternatives? What was the observed impact of these decisions in the enterprise architecture?

By interviewing the stakeholders we understood the context which influenced their decision making: During the execution of the enterprise transformation another high level decision from the Luxembourgish government had to be applied in the organization. The government decided that the RTO had to be merged with another national research and technology institution. This implied the need for serious changes in the organizational structure since some departments of the 


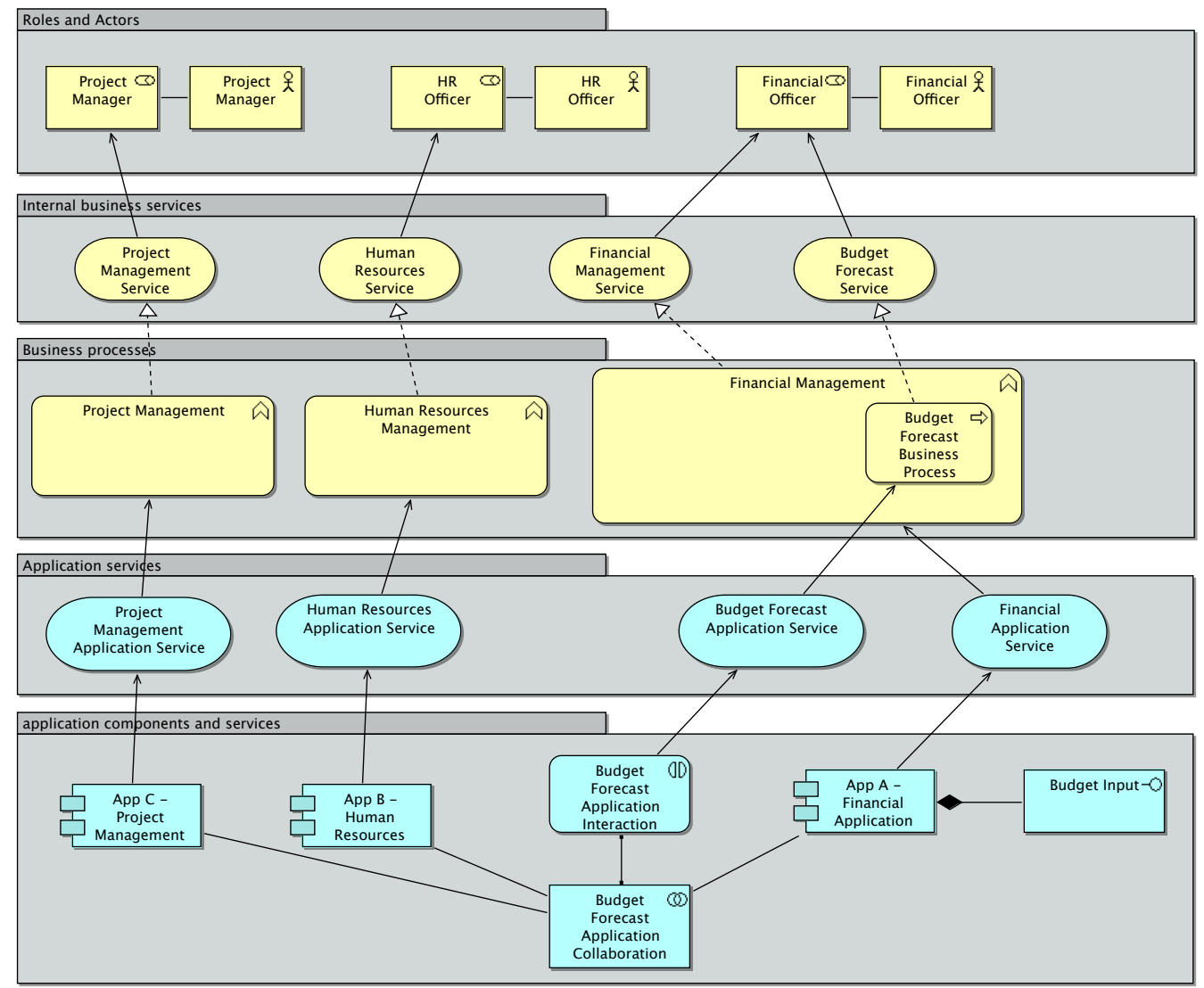

Fig. 3. To be architecture - Second iteration

RTO had overlapping roles with departments of the other organization. Moreover new business models should be defined based on the exchange of research expertise of research groups.

The upcoming merge of the organization posed some serious design challenges on the involved stakeholders of the budget estimation business process. On the one hand they had ambitious design goals considering the realization of this business need, while on the other hand they had to compromise because of the merge. It was not clear how the financial departments and business processes would be merged, therefore the risk of wasting budget for significant business and IT development was high.

Despite the fact that the initial plan of the stakeholders was the acquisition of a new COTS application, budget and time restrictions led stakeholders to the decision of the upgrade and collaboration of the in house applications. Several issues occurred after the execution of these decisions. As an example, the deci- 
sion for a spreadsheet template that would facilitate users to enter budget data was problematic. What occurred was that each department start modifying the template and the order of data. The financial officers that had to collect and process this data started having problems during the execution of the budget forecast business process.

Without rationalization the above reasons behind the architecture designs in Figs 1-3 remain implicit. Yet clearly such rationalization is useful. For example: by using rationalization one explicates the negative observed impact of diverging spreadsheets as a result of the introduction of the new business process As a result this negative observed impact can be anticipated on for future similar decisions. In the next section we present the objectives of an enterprise architecture rationale management system.

\section{Objectives of an enterprise architecture rationale management system}

As already discussed, design rationale is a well established domain and a plethora of approaches have been developed in the area of Software Architecture. However, the domain of Enterprise Architecture still remains unexplored. In a survey [6] that was conducted among 65 enterprise architecture practitioners, it was indicated that rarely they document their design decisions in a standardized way. Instead of a standardized method they capture rationale information by using word processors and other unstructured methodologies. Possibly this lack of a standardized way of documenting and using rationale information in enterprise architecture discourages practitioners from capturing this information. Motivated by the case study we discuss what are the challenges and potential objectives of an design rationale approach for Enterprise Architecture.

Challenge 1: One of the main goals of Enterprise architecture is the provision of reasoning of design decisions. However, the capturing of reasoning during an enterprise architecture design process is challenging due to the fact that the design decisions have to satisfy the requirements of stakeholders from different domains (Business, IT). As we previously saw in our case study, different factors influence their decision making, which in turn affect the quality of design decisions. Situations like time stress, budget restrictions or the conformance with organizational principles are common in enterprise architecture and change the way that stakeholders make design decisions. The objective for enterprise architecture is the capturing of this reasoning process. In doing so, stakeholders which analyze EA designs will be capable to better understand why specific decisions were made and under which decision making context.

Challenge 2: Another important aspect of design rationale approaches is the business/IT alignment. This means that the IT artifacts should support effectively the realization of new business processes. As we saw, during an enterprise transformation several design decisions are made, both in Business and IT sides. 
These design decisions rarely exist in isolation. Rather, design decisions are often cross cutting and intertwined. A rationale management system for enterprise architecture should be able to capture the different types of relationships among design decisions. For example, design decisions made on business levels can infer design decisions in IT level of the organization and vice-versa. On the other hand design decisions can be interrelated with a specific EA Artifact or domain of the Enterprise. An RMS system should be able to distinguish between these different relationships of design decisions. In this way it would better express the traceability and dependencies of design decisions in enterprise architecture.

Challenge 3: Another important objective is the traceability between the enterprise architecture design and design decisions. More specifically, during the analysis of the enterprise design, stakeholders should be able to identify which design decisions constitute specific EA artifacts and how the design decisions of these artifacts are related with other design decisions in the architecture. For example, we can start tracing from the addition of the new application interface of the financial application and we can check which decisions are related with this artifact.

Challenge 4: Sometimes design decisions can have unanticipated consequences in the artifact itself or in different artifacts in the enterprise architecture. As an example, consider the design decision for the spreadsheet template that had negative consequences in the use of a budget forecast business process. A rationale management system should also keep track of these incidents and how they were addressed by means of newer decisions. By doing so enterprise architects who want to analyze and have a holistic view of the architecture can identify existing vulnerabilities in the enterprise which can be prevented by remaking the same design decision in future architectural transformations.

\section{Research Questions}

In this section we define a set of concrete research questions. We argue that these research questions can address the above-mentioned objectives and in turn the challenges for a rationale management system for enterprise architecture.

Question 1: Which are the essential decision details concepts that rationalize design decisions in Enterprise Architectures?

There is a plethora of approaches for the rationalization of different domains (civil, software architecture). These approaches have introduced different sets of domain specific concepts. By answering this research question we aim to identify which concepts from existing frameworks can be used for the domain of enterprise architecture and what is actually missing to provide a holistic overview of design rationale in enterprise architecture. The identification of these concepts will provide a taxonomy of rationalization information for enterprise architecture and in turn the basis for the development of a design theory for the same 
purpose. The challenge, from a design theory point of view, is the identification of possible relationships among these concepts which in turn will enhance the utility of the design theory.

Question 2: How do we make explicit the underlying decision making process that was executed during the EA design?

As it was also stated, the decision making environment in Enterprise Architecture is challenging due to the implication of different stakeholders from different domains and due to factors that affect the decision making process. We argue that the capturing and representation of this underlying information can assist stakeholders during the inspection of the as-is architecture to analyze the evaluation process for specific decisions and recognize which factors actually influenced this decision making process. By doing so, they can consult for their future evaluations by following/avoiding good/bad evaluations from past decisions making processes.

Question 3: How do we capture and represent different decision relationships in Enterprise Architecture?

The confrontation of this research question will provide a holistic overview and traceability of the enterprise architecture. The domain of enterprise architecture introduces several challenges since decisions from a specific domain (IT) can be related with decisions of the same or another domain (Business). The same applies also for the outcomes of EA decisions since the application of a specific decision may introduce problematic situations in different domains of the enterprise. To cope with this research question we will investigate existing design rationale approaches from different domains and we will identify the specificities imposed by the domain of enterprise architecture.

\section{Conclusions and future work}

In this paper, based on a case study in a Luxembourgish research and Technology organization we identified the importance of capturing design rationale information. Moreover, motivated by this case study we discussed what were the objectives for the development of a rationale management system for an enterprise architecture and we provided a list of concrete research questions which can be used for the development of such a framework.

For future research, we aim to apply the EA Anamnesis approach to the RTO case study and evaluate its capability to capture the underlying design rationale knowledge in the context of an enterprise transformation. Based on the derived objectives and research questions, we aim to further improve our existing approach by means of metamodel modifications.

\section{References}

1. Lankhorst, M., et al.: Enterprise Architecture at Work: Modelling, Communication and Analysis. 3rd edn. Springer Publishing Company, Incorporated (2012) 
2. Op 't Land, M., Proper, H., Waage, M., Cloo, J., Steghuis, C.: Enterprise Architecture - Creating Value by Informed Governance. Springer, Berlin, Germany (2008)

3. Lagerström, R., Johnson, P., Höök, D.: Architecture analysis of enterprise systems modifiability-models, analysis, and validation. Journal of Systems and Software $\mathbf{8 3}$ (2010) 1387-1403

4. Feltus, C., Dubois, E., Proper, E., Band, I., Petit, M.: Enhancing the archimate standard with a responsibility modeling language for access rights management. In: Proceedings of the Fifth International Conference on Security of Information and Networks. SIN '12, New York, NY, USA, ACM (2012) 12-19

5. Tang, A., Jin, Y., Han, J.: A rationale-based architecture model for design traceability and reasoning. Journal of Systems and Software 80 (2007) 918 - 934

6. Plataniotis, G., de Kinderen, S., van der Linden, D., Greefhorst, D., Proper, H.A.: An empirical evaluation of design decision concepts in enterprise architecture. In: Proceedings of the 6th IFIP WG 8.1 working conference on the Practice of Enterprise Modeling (PoEM 2013). (2013)

7. Plataniotis, G., de Kinderen, S., Proper, H.A.: Ea anamnesis: An approach for decision making analysis in enterprise architecture. International Journal of Information System Modeling and Design (IJISMD) (2014)

8. The Open Group: ArchiMate 2.0 Specification. Van Haren Publishing (2012)

9. Runeson, P., Hst, M.: Guidelines for conducting and reporting case study research in software engineering. Empirical Software Engineering 14 (2009) 131-164 